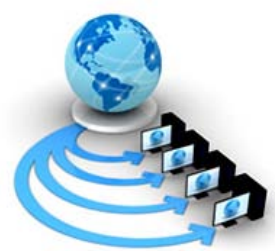

Volume 9, No. 3, May-June 2018

International Journal of Advanced Research in Computer Science

RESEARCH PAPER

\title{
DETERMINING A FLEXIBLE LOW-RANK GRAPH USING SUBSPACE CLUSTERING
}

\author{
J.N.CHANDRA SEKHAR \\ Assistant Professor, Department of CSE, \\ KKR \& KSR Institute of Technology and Sciences,India
}

\begin{abstract}
By utilizing a meager representation or low-rank representation of information, chart based subspace bunching has as of late pulled in impressive consideration in PC vision, given its ability and productivity in grouping information. Be that as it may, the chart weights worked by utilizing representation coefficients are not the correct ones as the conventional definition. The two stages of portrayal what's more, bunching are led in an autonomous way; in this manner a general ideal outcome can't be ensured. Moreover, it is vague how the bunching execution will be influenced by utilizing this chart. For instance, the diagram parameters, i.e., the weights on edges, must be falsely pre-indicated while it is extremely hard to pick the ideal. To this end, in this paper, a novel subspace bunching by means of taking in a versatile low-rank diagram proclivity lattice is proposed, where the partiality framework and the representation coefficients are found out in a bound together system. All things considered, the pre-figured chart regularize is successfully blocked and better execution can be accomplished. Test comes about on a few well known databases illustrate that the proposed technique performs better against the state-of the- workmanship approaches, in grouping.
\end{abstract}

Keywords - inadequate portrayal, low-rank portrayal, subspace grouping, versatile low-rank diagram, partiality grid.

\section{INTRODUCTION}

In reality, high dimensional information are pervasive for numerous applications, for example, picture preparing. The current work of subspace bunching has pulled in impressive consideration because of its ability and effectiveness in information bunching [1]. The hidden suspicion is that the watched information nearly lie in/close to some lowdimensional subspaces [2]. Profited from a pairwise comparability diagram, information grouping is promptly changed into a chart segment issue [3][4][5][6].

Typically, an enlightening chart,regardless of coordinated or undirected, assumes a key part for those chart based calculations intended with the end goal of information grouping, subspace learning, and so on. In this manner, to develop chart, the most widely recognized way is to process removes on the crude information straightforwardly, e.g., kNearest- Neighbor utilizing cosine or warmth portion separations [7]. On the other hand, the diagram edge weights can likewise be gotten by limiting '2-standard of the reproduction blunder in a neighborhood fix [8]. Besides, Nie et al. proposed taking in the likeness lattice by adaptively allotting neighbors for every datum point [9]. To accomplish the genuine diagram structure of information, they too proposed a compelled Laplacian rank (CLR) minimization issue to take in a chart with precisely $\mathrm{k}$ associated parts, where $\mathrm{k}$ is the correct number of groups [10].

Nonetheless, the chart worked from the portrayal coefficients utilizing the above methodologies isn't precisely the one as the customary definition is deterministically. Besides, it isn't clear how bunching execution will be influenced by utilizing this diagram. In the above strategies, the diagram development process is regularly isolated into two stages. For the most part, the initial step is to build a symmetric proclivity lattice by utilizing the educated low- rank or scanty coefficients [11][12]. Information focuses I and $\mathrm{j}$ are in a similar subspace if their affinities $\mathrm{Wij}=$ $\mathrm{Wji} \lambda=0$. Else, they are in various subspace. Next, a weighted undirected chart is constructed utilizing information focuses as hubs and the liking Wij as weights on edges. To some

degree, the diagram development and learning portrayal coefficients of information are intentionally isolated for

simple taking care of. In any case, this process may bring about two disadvantages. One is that the scholarly diagram does not have the first definition. The other is that it might prompt a traded off bunching execution. In spite of the fact that the diagram regularized inadequate [13] or low-rank [14][4] subspace bunching technique can accomplish great outcomes, Wij must be precomputed which may not mirror the genuine closeness between information focuses. That is, two focuses, lying in various subspaces (e.g., close to the crossing point of two subspaces), could be extremely near each other, and the other way around. Subsequently, the educated portrayal of information may bungle the genuine proclivity between information focuses. Therefore, grouping execution is then traded off.

The principle commitments in our paper are condensed underneath.

1) To our best information, it is the principal endeavor to incorporate low-rank portrayal and diagram development in a bound together structure. It is altogether not quite the same as past works that takes in the low-rank portrayal and after that build the diagram framework, in an isolated way.

2) In light of the property of intra-subspace projection strength [15], we propose to vanish some insignificant values in the proclivity network for strength. All things considered, the adapted "great" proclivity network 
will significantly manage and improve the learning of the coefficients, and the other way around.

3) We apply our proposed strategy to a few bunching errands including unsupervised way and semiregulated way.

\section{RELATED WORK}

\section{A. Scanty Subspace Clustering}

Scanty portrayal [16], which has been ended up being a fruitful device for speaking to and compacting highdimensional signals, gives a measurable model to discovering effective and minimized flag portrayals. On account of this helpful instrument, the standard SSC calculation [17] has been proposed to group information focuses that lie in an association of low-dimensional. Consider the case that data $\mathrm{X}=[\mathrm{x} 1 ; \mathrm{x} 2 ;$ :::; $\mathrm{xN}] \mathrm{Rd} \_\mathrm{S} \mathrm{N}$ are drawn from a union of multiple subspaces given by ki=1 $\mathrm{Si}$, where S1, S2, ..., Sk are low-dimensional subspaces. By exploiting the self-expressiveness property of the data, the formulation of SSC becomes

$$
\min _{Z}\|Z\|_{1}+\lambda\|E\|_{F}^{2} \text {, s.t. } X=X Z+E, \operatorname{diag}(\mathrm{Z})=0 \text {. }
$$

Subspaces.As for the scanty coefficients network Z, there are two translations all in all. Initially, we can clarify every section zi of $\mathrm{Z}$ as another portrayal of the information point xi as far as different examples in X. a portrayal for xi. Furthermore, the component Zij mirrors the "closeness" between information match $\mathrm{xi}$ and $\mathrm{xj}$, subsequently $\mathrm{Z}$ is at times called a proclivity lattice. For the second issue, a bunching calculation, for example, K-means can be in this manner connected to $\mathrm{Z}$ for definite division. This is a typical system in ghostly procedure based subspace bunching.

\section{B. Low-Rank Representation}

LRR technique [7][18] goes for looking for the most reduced rank portrayal of allinformation together so every one of information point can be spoken to as a straight mix of a few bases. The issues including the estimation of low-rank networks have attracted extensive consideration late years [14][4][16]. The low-rank regularize in LRR has a profound connection with the current hypothetical advances on hearty primary segment examination (RPCA) [5][6], which prompts new and intense demonstrating alternatives for some applications. It has been shown that LRR can viably catch the fundamental low-dimensionalStructures of information.

\section{Subspace Clustering by means of Sparse Prior}

As of late, enlivened by the advances of SSC and LRR, numerous chart based subspace grouping calculations have been developed[13][19][4][14]. For instance, to protect the complex structure of information, Cai et. al.[13] proposed a diagram regularized inadequate coding. Yin et. al. fused Laplacain diagram requirements into the standard LRR model to safeguard the geometric data from single side [14] and twoside [4] viewpoint. When all is said in done, this kind of subspace bunching can be assembled into ghostly grouping based strategies, which have been exhibited to perform extremely well for some applications in PC vision [19]. On a fundamental level, this sort of subspace bunching calculations comprises of two stages. The initial step is taking SSC (or LRR) type ways to deal with take in another portrayal of information by means of self-expressiveness property. In principle, focuses $\mathrm{I}$ and $\mathrm{j}$ will be drawn from a similar subspace if Wij 1 , and this is false when Wij 0 . Next, a weighted undirected chart is constructed in view of this symmetric partiality framework, where information focuses are the hubs and the affinities Wij are weights on the edges of the diagram. Information bunching result is then acquired by gathering the eigenvectors of the chart Laplacian through focal grouping systems, for example, K-means. According to the above portrayal, we can see that the most basic advance is to build a "decent" liking network to ideally mirror the genuine pairwise connection between information focuses. Besides, the development of proclivity framework and representation learning are isolated. In chart installing [20], if two data focuses are shut in the feeling of the characteristic geometry, their inserting/mappings in another space must be near each other. Persuaded by this supposition, we built up a few diagram regularized low-rank subspace grouping strategies [14][4][21]. In science, this closeness safeguarding can be figured as,

$$
\min _{Z} \sum_{i j}\left\|\mathbf{z}_{i}-\mathbf{z}_{j}\right\|^{2} b_{i j}=\frac{1}{2} \operatorname{tr}\left(Z^{T} L_{B} Z\right),
$$

\section{THE PROPOSED METHOD}

We propose an integrated model of low-rank subspace clustering.

\section{A. Adaptive graph structure learning}

Considering data $\mathrm{X}=[\mathrm{x} 1 ; \mathrm{x} 2 ;: .: ; \mathrm{xN}]$, the i-th data point $x i$ is connected to other points with probability $\operatorname{bij}(j=$ 1 .....n) as a neighbor.

Motivated by the progress of complex learning [1][10], it is an instinctive thought that the information focuses being close have comparable portrayal coefficients [16][11]. All things considered, the inherent nearby structure of information is safeguarded. In particular, if xi and xj are near each other in the first space, at that point their portrayals zi and zj ought to be close as well. In light of this perception, to decide the likelihood bij , the accompanying issue is frequently fathomed.

$$
\min _{b_{i j}} \sum_{i j}\left\|\mathbf{z}_{i}-\mathbf{z}_{j}\right\|_{2}^{2} b_{i j}, \text { s.t. } \mathbf{b}_{i}^{T} \mathbf{1}=1,0 \leq \mathbf{b}_{i} \leq 1 \text {, }
$$

Moreover, to cure the inadequacies of the current techniques, our strategy is proposed to understand the accompanying rank minimization issue.

$$
\min _{Z, E,\left\{b_{i j}\right\}} \lambda_{1} \operatorname{rank}(Z)+\lambda_{2} \sum_{i j}\left\|\mathbf{z}_{i}-\mathbf{z}_{j}\right\|_{2}^{2} b_{i j}+\lambda_{3} \sum_{i j} b_{i j}^{2}+\Phi(E) .
$$

\section{B. Convergence and complexity analysis}


The advancement methodology clarified in past subsection is a regular square shrewd organize drop connected to piecemulti-raised capacity. Definite meeting examination can be found in [22]. We have to call attention to that the improvement issue isn't raised and consequently there might be limitlessly numerous nearby minima and the last outcomes rely upon instatement. To obviously demonstrate the merging of our SC-LALRG, we demonstrate the bends of the cost (i.e., log-estimation of target work) versus cycles on Extended YaleB and COIL20, separately, in Fig. 1.

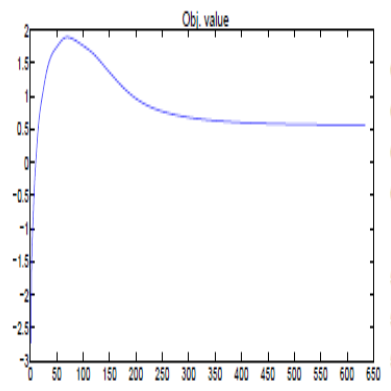

(a)

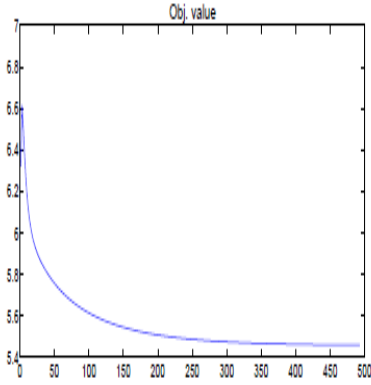

Fig. 1: Convergence curve of our proposed algorithm on (a) Extended YaleB and (b) COLL20. Note that a tearly iterations the variables are not feasible, although they have lower cossts. When it converges, the variables are feasible.

What's more, to survey the many-sided quality of the proposed calculation, we not just give the multifaceted nature of the proposed calculation spoke to by $\mathrm{O}(\mathrm{n})$, yet in addition list every cycle of the calculation activity conditions 1, as appeared in Table II.

\section{TABLE II: Complexity analysis of the proposed algorithm.}

\begin{tabular}{l||l|l|l}
\hline & Addition & Multiplication & Complexity \\
\hline Update E & $d n+n^{2}$ & $d n^{2}+d^{2} n+d n$ & $\mathcal{O}\left(d n^{2}\right)$ or $\mathcal{O}\left(d^{2} n\right)$ \\
Update Z & $d n+n^{2}$ & $r n^{2}$ & $\mathcal{O}\left(m n^{2}\right)$ \\
Update Q & $d n+n^{2}$ & $d n^{2}+d n+n^{2.373}$ & $\mathcal{O}\left(d n^{2}\right)$ \\
Update B & $n^{2}$ & $d n+n^{2}$ & $\mathcal{O}\left(n^{2}\right)$ \\
\hline
\end{tabular}

\section{EXPERIMENTAL RESULTS}

To check the viability of our proposed strategy, in this area, we led a few test tests on engineeredinformation and genuine databases, for example, Extended YaleB, ORL, COIL20 and MNIST.

\section{A. Manufactured information}

Initial, manufactured information is utilized to assess the bunching productivity of the proposed SC-LALRG. The manufactured information isarbitrarily produced in fivedimensional space, which incorporates 500 examples altogether. To start with we create three arrangements of 50, 150 also, 300 boisterous information in three round districts, individually, in the hyper plane of the initial two dimensional subspace in the 5 dimensional space. Locally information is in applicable subspaces. To imitate 5 dimensional information, we additionally include clamor in the other three measurements. That is, the initial two measurements of information comprise of a three-hover shape as appeared in Fig. 2(a) while the rest three measurements are commotions. Not surprisingly, Gaussian clamor have been utilized as a part of our examination. As just the initial two measurements contain helpful data, the bunching undertaking is testing. The bunching aftereffect of SC-LALRG is exhibited in Fig. 2 where it is exhibited that SC-LALRG can recoup the subspace adequately, which is nearly the same as the subspace framed by the initial two critical measurements, seeing Fig. 2 (b) and (c).

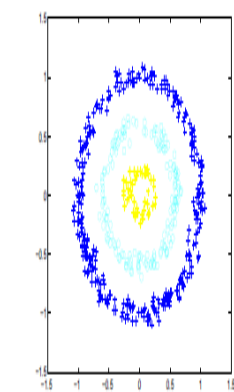

(1)

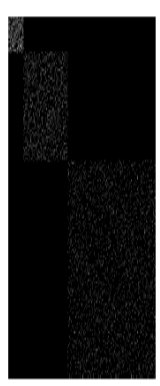

(b)

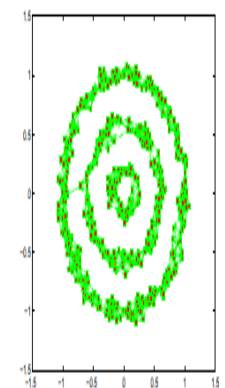

$(d)$

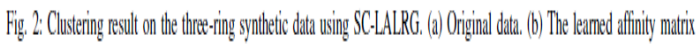
(Bess seen in lagere resolution). (c) The leamed grapht.

\section{B. Affectability to Parameters}

There are a few regularization parameters and the extent of neighborhood $\mathrm{K}$ influencing the execution of SC-LALRG. In the accompanying analyses, we think about the impact of parameters and K on COIL20 with 20 objects. By utilizing diverse estimations of the parameters, the inconstancy of SC-LALRG grouping execution is analyzed. In particular, $\mathrm{K}$ is fluctuating from 2 to 50 while $\lambda$ is settled to $1 \mathrm{e}-4$. The estimation of $\lambda$ is looked over the set f1e-5, 1e-3, 1e-2, 1e-1, $1,2,5,10 \mathrm{~g}$ when settling $\mathrm{K}$ to be 10 . The comparing impacts as far as ACC are given in Fig. 7, from which we can see that our strategy performs well and steadily when $\lambda$ $10^{-3}$. At that point, we can decide the $\mathrm{K}$ and $\lambda$ at first. Besides, to completely research the affectability to parameters, we test the proposed strategy by fluctuating $\lambda 1$ and $\lambda 2$ as their significance to the grouping execution. By settling $\lambda 3=0: 05$, the grouping execution, fluctuating with $\lambda 1$ and $\lambda 2$, are given in Fig. 8. $\lambda 1$ goes over f1e-5, 1e-3, 5e$3,1 \mathrm{e}-2 \mathrm{~g}$ and $\lambda 2$ fluctuates with f1e-5, 1e-4, 1e-3, 5e-3, 1e-2, $1 \mathrm{e}-1,5 \mathrm{e}-1,1 \mathrm{~g}$. As can be seen, the execution, as far as ACC and NMI, are steady. This approves essentially setting the parameters to be equivalent is sensible. 

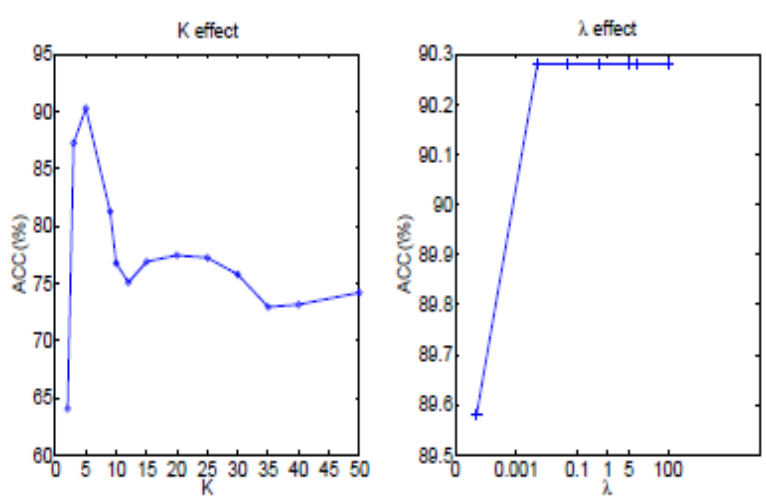

Fig. 7: The effects of parameters on COIL20 database. (a) $\mathrm{K}$ effect, (b) $\lambda$.

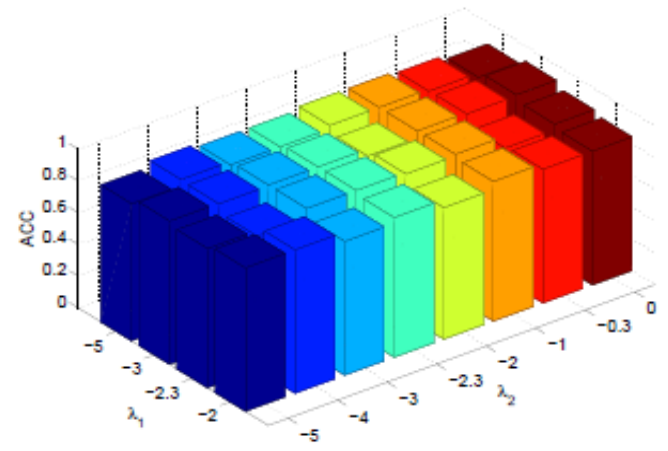

(a) $\mathrm{ACC}$

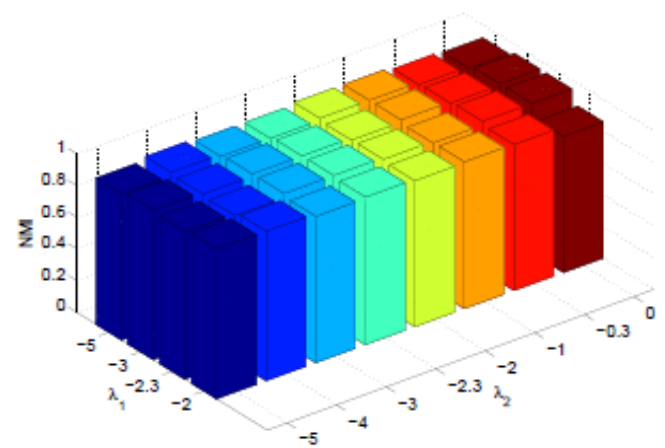

(b) NMI

Fig. 8: The effects of $\lambda_{1}$ and $\lambda_{2}$ (log-value) on COIL20 database.

\section{CONCLUSION}

In this paper a novel subspace bunching by means of learning a versatile low-rank diagram is proposed, where the partiality lattice and portrayal coefficients are mutually learned in a brought together structure. It cannot just join the low rank portrayal into chart development process, yet additionally find the worldwide blend of subspaces structure inside information. Exploratory outcomes, in the unsupervised and semi-managed learning ways, demonstrate that the proposed strategy can give preferable grouping arrangements over the cutting edge approaches because of a mix structure.

\section{REFERENCES}

[1] R. Vidal. Subspace clustering. IEEE Signal Processing Magazine, 28(2):52-68, 2011.

[2] Lance Parsons, EhteshamHaque, and Huan Liu. Subspace clustering for high dimensional data: A review. SIGKDD Explorations Newsletter, 6(1):90-105, June 2004.

[3] Jianbo Shi and Jitendra Malik.Normalized cuts and image segmentation. IEEE Transactions on Pattern Analysis and Machine Intelligence, 22:888-905, 1997.

[4] Ming Yin, JunbinGao, Zhouchen Lin, Qinfeng Shi, and Yi Guo. Dual graph regularized latent low-rank representation for subspace clustering. IEEE Transactions on Image Processing, 24(12):4918-4933, 2015.

[5] Ran He, Liang Wang, Zhenan Sun, Yingya Zhang and Bo Li. Information theoretic subspace clustering. IEEE Transactions on Neural Networks and Learning Systems, 27(12):2643-2655,2016.

[6] HongchangGao, FeipingNie, Xuelong Li, and Heng Huang. Multiview subspace clustering.In 2015 IEEE International Conference on Computer Vision (ICCV), pages 42384246, 2015.

[7] Mikhail Belkin and ParthaNiyogi.Laplacianeigenmaps for dimensionality reduction and data representation. Neural Computation, 15(6):1373-1396, 2003.

[8] S. T. Roweis and L. K. Saul.Nonlinear dimensionality reduction by locally linear embedding. Science, 290:2323-2326, 2000.

[9] FeipingNie, Xiaoqian Wang, and Heng Huang. Clustering and projected clustering with adaptive neighbors. In Proceedings of the 20th ACM SIGKDD International Conference on Knowledge Discovery and Data Mining, pages 977-986, 2014.

[10] FeipingNie, Xiaoqian Wang, Michael I. Jordan, and Heng Huang.The constrained laplacian rank algorithm for graph-based clustering.In Proceedings of AAAI 2016, pages 1969-1976, 2016.

EhsanElhamifar and Ren_e Vidal. Sparse subspace clustering: Algorithm, theory, and applications. IEEE Transactions on Pattern Analysis and Machine Intelligence, 35(11):2765-2781, 2013.

[12] Guangcan Liu, Zhouchen Lin, Shuicheng Yan, Ju Sun, and Yi Ma. Robust recovery of subspace structures by lowrank representation. IEEE Transactions on Pattern Analysis and Machince Intelligence, 35(1):171 - 184, Jan. 2013.

[13] Miao Zheng, Jiajun Bu, Chun Chen, Can Wang, Lijun Zhang, GuangQiu, and Deng Cai. Graph regularized sparse coding for image representation. IEEE Transactions on Image Processing, 20(5):1327-1336, 2011.

[14] Ming Yin, JunbinGao, and Zhouchen Lin. Laplacian regularized lowrank representation and its applications. IEEE Transactions on Pattern Analysis and Machine Intelligence, 38(3):504-517, 2016.

[15] Xi Peng, Zhang Yi, and Huajin Tang. Robust subspace clustering via thresholding ridge regression. In Proceedings of AAAI, AAAI'15, pages 3827-3833, 2015.

[16] D.L. Donoho, M. Elad, and V. Temlyakov. Stable recovery of sparse overcomplete representations in the presence of noise. IEEE Transactions on Information Theory, 52(1):618, 2006.

[17] D. L. Donoho. Compressed sensing. IEEE Transcations on Information Theory, 52(4):1289-1306, 2006.

[18] Guangcan Liu, Zhouchen Lin, Shuicheng Yan, Ju Sun, and Yi Ma. Robust recovery of subspace structures by lowrank representation. IEEE Transactions on Pattern Analysis and Machince Intelligence, 35(1):171 - 184, Jan. 2013. 
[19] Ren_e Vidal and Paolo Favaro. Low rank subspace clustering (LRSC). Pattern Recognition Letters, 43(1):4761, 2014.

[20] Shuicheng Yan, Dong Xu, Benyu Zhang, Hong-Jiang Zhang, Qiang Yang, and Stephen Lin. Graph embedding and extensions: A general framework for dimensionality reduction. IEEE Transactions on Pattern Analysis and Machince Intelligence, 29(1):40-51, 2007.
[21] Ming Yin, JunbinGao, and Yi Guo. Nonlinear low-rank representation on Stiefel manifolds. Electronics Letters, 51(10):749-751, 2015.

[22] YangyangXu and Wotao Yin.A block coordinate descent method for regularized multiconvex optimization with applications to nonnegative tensor factorization and completion. SIAM Journal of Imaging Sciences, 6(3):1758-1789, 2013. 